\title{
Laminar Patterning in the Developing Neocortex by Temporally Coordinated Fibroblast Growth Factor Signaling
}

\author{
Hiroshi Hasegawa, ${ }^{1}$ Shizuko Ashigaki, ${ }^{1}$ Masako Takamatsu, ${ }^{1}$ Rika Suzuki-Migishima, ${ }^{2}$ Norihiko Ohbayashi, ${ }^{3}$ \\ Nobuyuki Itoh, ${ }^{4}$ Shinji Takada, ${ }^{3}$ and Yasuto Tanabe ${ }^{1}$ \\ ${ }^{1}$ Neural Organization Research Team, Mitsubishi Kagaku Institute of Life Sciences, Precursory Research for Embryonic Science and Technology, Japan Science and \\ Technology, ${ }^{2}$ Mouse Genome Technology Center, Machida, Tokyo 194-8511, Japan, ${ }^{3}$ Center for Integrative Bioscience, Okazaki National Research Institutes, \\ Myodaiji-cho, Okazaki 444-8585, Japan, and ${ }^{4}$ Department of Genetic Biochemistry, Kyoto University Graduate School of Pharmaceutical Sciences, Yoshida- \\ Shimoadachi, Sakyo, Kyoto 606-8501, Japan
}

Laminar organization, a fundamental neural architecture in the CNS, is a prominent feature of the neocortex, where the cortical neurons in spatially distinct layers are generated from the common progenitors in a temporally distinct manner during development. Despite many advances in the characterization of the molecular mechanisms of the radial migration of cortical neurons, the way in which the early-late temporal sequence of cortical neuron generation is linked with the deep-superficial spatial sequence of cell body positioning remains obscure. Using in vivo electroporation-mediated gene transfer, we show here that the activities mediated by fibroblast growth factor receptors (FGFRs) in cortical progenitors are critical for conferring proper migratory properties on nascent neuronal progeny. Furthermore, we provide supportive evidence that Pea3 subfamily members of Ets (Pea3-Ets) transcription factors mediate the activities of FGFR at the mid to late phase of neocortical development. In addition, using FGF18 knock-out mice, we demonstrate that FGF18 expressed by early-generated cortical neurons in the cortical plate is critical for the expression of Pea3-Ets transcription factors and that FGF18 is sufficient to induce their expressions. Our results thus imply that a feedback mechanism mediated by FGF signaling is involved in setting up the proper laminar positioning of cortical neurons; FGF18 derived from early-generated cortical neurons acts on the cortical progenitors expressing FGFRs and induces the expression of Pea3-Ets transcription factors that, in turn, confer proper migratory behaviors on nascent cortical progeny during the mid to late stages of neocortical development.

Key words: laminar patterning; neocortex; radial migration; feedback control; fibroblast growth factor; Ets transcription factors

\section{Introduction}

In the developing neocortex, hierarchical series of radial and tangential migration of distinct neuronal subtypes culminate in the laminar organization of distinct cortical areas that encode a particular set of brain functions (Anderson et al., 2002; Gupta et al., 2002; Nadarajah and Parnavelas, 2002; Olson and Walsh, 2002). Cortical neurons located in distinct layers are generated from common progenitors in the underlying ventricular zone (VZ) in a temporally precise manner. These cells migrate in a radial direction to comprise the cortical plate (CP) in an "inside-out" manner; the early-generated cortical neurons are located in deep layers, whereas the late-generated cortical neurons migrate beyond the early-generated cortical neurons and are located in up-

\footnotetext{
Received Dec. 28, 2003; revised Aug. 23, 2004; accepted Aug. 23, 2004.

This work was supported by grants from Precursory Research for Embryonic Science and Technology of the Japan Science and Technology. We thank T. Saito (Kyoto University) for his kind advice on the electroporation techniques, H. Yamazaki (Kyoto University) for his kind advice on the in situ hybridization techniques, J. Miyazaki (Osaka University) for pCAGGS, H. Ohuchi (Tokushima University) for FGF8, and Y. Arimatsu (Mitsubishi Kagaku Institute of Life Sciences) for anti- $\beta$ III tubulin antibody. We are grateful to T. M. Jessell (Columbia University) for his initial encouragement of this work.

Correspondence should be addressed to either of the following: Yasuto Tanabe, 11 Minami-0oya, Machida Tokyo, 194-8511, Japan, E-mail: ytanabe@libra.Is.m-kagaku.co.jp; or Shinji Takada, 5-1 Higashiyama, Myodaijicho, 0kazaki, 444-8585, Japan, E-mail: stakada@nibb.ac.jp.

DOI:10.1523/JNEUROSCI.3070-04.2004

Copyright $\odot 2004$ Society for Neuroscience $\quad$ 0270-6474/04/248711-09\$15.00/0
}

per layers. Previous studies using a heterochronic transplantation approach suggested that the specification of laminar identities of cortical neurons is controlled by environmental signals that cortical progenitors encounter at around the time of their final cell division (McConnell and Kaznowski, 1991; McConnell, 1995). These studies led to the suggestion that these environmental factors affect cortical progenitors in a feedback manner to trigger the temporal switch for the specification of distinct laminar identities. In contrast, the mechanism by which distinct radial migratory behaviors and laminar allocation of cortical neurons is controlled is poorly understood (Marin and Rubenstein, 2003). Moreover, the nature of cell-extrinsic signals, the way they act on cortical neurons, and cell-intrinsic programs that in turn control proper migration and cell body allocation remain to be defined.

Members of Ets family transcription factors have been implicated in the control of development of a wide variety of tissues (Wasylyk et al., 1998). They hold a winged-helix-turn-helix structure similar to that of hepatocyte nuclear factor $3 /$ forkhead transcription factors and are critical downstream nuclear targets of signal transduction cascades. In the developing spinal cord, three Pea3 subfamily members of Ets (Pea3-Ets) transcription factors, Erm, Er81, and Pea3, are expressed by restricted groups of cells (Lin et al., 1998). The expression of Pea3-Ets transcription factors in these cells is induced by cell-extrinsic signals derived 
from the periphery and is critical for the control of proper cell body positioning and axonal trajectory patterns (Lin et al., 1998; Arber et al., 2000; Haase et al., 2002; Livet et al., 2002; Helmbacher et al., 2003; Patel et al., 2003). These observations led us to examine whether Pea3-Ets transcription factors expressed in the developing neocortex (Chotteau-Lelievre et al., 1997) might be involved in the control of characteristic laminar framework formation.

We show here that Pea3-Ets transcription factors are expressed by cortical progenitors at the mid to late stages of neocortical development and appear to be critical for conferring proper migratory behaviors on nascent neuronal progeny. The expression of Pea3-Ets transcription factors depends on the activation of FGFRs in cortical progenitors and is controlled by FGF18 signals derived from early-generated cortical neurons in the CP. Our studies imply that temporally coordinated FGF signaling regulates distinct migratory behaviors and laminar allocation of cortical neurons at the mid to late stages of neocortical development and thereby underlie the laminar patterning of developing neocortex.

\section{Materials and Methods}

Animals. ICR Jcl mice (CLEA Japan, Tokyo, Japan) were mated to obtain offspring in-house in an environmentally controlled room according to the Guidelines for Use of Laboratory Animals (Japan Neuroscience Society) and the Animal Care and Use Committee (Mitsubishi Kagaku Institute of Life Sciences). Noon of the date on which the vaginal plug was detected in the morning was designated as embryonic day 0.5 (E0.5). Embryos of FGF18 knock-out mice were obtained as described previously (Ohbayashi et al., 2002).

In situ hybridization and immunohistochemistry. Embryos were fixed by cardiac perfusion with $4 \%$ paraformaldehyde (PFA) in $0.1 \mathrm{M}$ phosphate buffer $(\mathrm{PB}), \mathrm{pH} 7.3$, and postfixed in the same buffer overnight at $4^{\circ} \mathrm{C}$. They were then placed in PBS with $25-30 \%$ sucrose for at least $24 \mathrm{hr}$ before embedding in OCT compound (Sakura, Tokyo). Frozen sections were cut with a cryostat (MICROM HM500OM, Carl Zeiss, Jena, Germany) at $10 \mu \mathrm{m}$ and mounted onto MAS-coated glass slides (Matsunami, Osaka, Japan).

In situ hybridization was performed essentially as described (Ishibashi et al., 1995), with minor modifications. Sections were first immersed in PBT (PBS containing $0.1 \%$ of Tween 20 ), treated with $1 \mu \mathrm{g} / \mathrm{ml}$ proteinase $\mathrm{K}$ in $\mathrm{PBT}$ at $37^{\circ} \mathrm{C}$ for $7 \mathrm{~min}$, and washed three times with PBT. They were subsequently fixed with 4\% PFA in PBS at room temperature for $30 \mathrm{~min}$ and again washed three times with PBT. Hybridization was performed overnight at $65^{\circ} \mathrm{C}$ with either digoxigenin-labeled or ${ }^{33} \mathrm{P}$-labeled $/{ }^{35} \mathrm{~S}$ labeled RNA probes. Hybridized sections were washed two times with buffer A [50\% formamide, $5 \times$ SSC $(150 \mathrm{~mm} \mathrm{NaCl}, 15 \mathrm{~mm}$ sodium citrate, $\mathrm{pH} 4.5)$ and $1 \% \mathrm{SDS}$ at $65^{\circ} \mathrm{C}$ for $30 \mathrm{~min}$ and then washed three times with buffer B ( $50 \%$ formamide, $2 \times \mathrm{SSC})$ at $65^{\circ} \mathrm{C}$ for $30 \mathrm{~min}$. For sections treated with digoxigenin-labeled RNA probes, sections were immersed in Tris-buffered saline (TBS), $\mathrm{pH} 7.0$, and TBS containing $0.1 \%$ Tween 20 (TBST), treated with 0.5\% Blocking Reagent (DIG Nucleic Acid Detection Kit; Roche, Mannheim, Germany), and incubated with alkaline phosphatase-conjugated anti-digoxigenin antibody (diluted to 1:1000; Roche) in TBST containing $0.5 \%$ Blocking Reagent at $4^{\circ} \mathrm{C}$ overnight. After washing three times with TBST containing $2 \mathrm{~mm}$ levamisol (Sigma, St. Louis, MO), they were treated with NTMT solution (100 mM Tris- $\mathrm{HCl}$, pH 9.5, $100 \mathrm{~mm} \mathrm{NaCl}, 50 \mathrm{~mm} \mathrm{MgCl}, 0.1 \%$ Tween 20, and 2 $\mathrm{mm}$ levamisol). The coloring reaction was performed in the NTMT solution containing $35 \mathrm{mg} / \mathrm{ml}$ nitro blue tetrazolium (Roche), $17.5 \mathrm{mg} / \mathrm{ml}$ 5-bromo-4-chloro-3-iodyl phosphate (Roche), and $2 \mathrm{~mm}$ levamisol. For sections treated with ${ }^{33} \mathrm{P}$-labeled or ${ }^{35} \mathrm{~S}$-labeled RNA probes, hybridized sections were washed in the same condition as described for the sections hybridized with digoxigenin-labeled RNA probes and then immersed in TBS. Sections were dehydrated in a series of ethanol solutions and exposed by dipping the slides in emulsion according to the manufacturer's protocol (Autoradiography Emulsion Type NTB-3; Kodak, New Haven,
CT). The dipped sections were kept at $4^{\circ} \mathrm{C}$ for 2-3 weeks and developed with HI-RENDOL/HI-RENFIX (Fuji Film, Tokyo, Japan).

The prepared RNA probes were Erm nucleotides 1385-2054 (Chotteau-Lelievre et al., 1997), Er81 nucleotides 1704-2854 (Brown and McKnight, 1992), Pea3 nucleotides 1491-2380 (Xin et al., 1992), FGFR3 nucleotides 3099-4070 (Ornitz and Leder, 1992), FGF18 nucleotides 403-922 (Hu et al., 1998), and FGF17 nucleotides 163-683 (Xu et al., 1999). RNA probes for FGFR1-3c were prepared as described previously (Ohbayashi et al., 2002).

Immunohistochemical staining was performed as described previously (Takiguchi-Hayashi et al., 2004). The primary antibodies (Abs) were polyclonal rabbit anti- $\beta$ III tubulin Ab (kindly provided by Dr. Arimatsu, Mitsubishi Kagaku Institute of Life Sciences) at 1:1000 and polyclonal rabbit anti-MAP2 Ab (Chemicon, Temecula, CA) at 1:200. The secondary $\mathrm{Ab}$ used was cyanine3 (Cy3)-conjugated anti-rabbit IgG (Jackson ImmunoResearch, West Grove, PA) at 1:200.

DNA constructs. A CAG promoter-driven EYFP (enhanced yellow fluorescent protein) expression vector was prepared by subcloning EYFP (Invitrogen, San Diego, CA) into a $p C A G G S$ vector (kindly provided by Dr. J. Miyazaki, Osaka University, Japan) (Niwa et al., 1991). A CAG promoter-driven FGF18 expression vector was prepared by subcloning FGF18 into a $p C A G G S$ vector. The dominant-negative (DN) Erm, Er81, and $P e a 3$ constructs were prepared by isolating the Ets domain of mouse Erm (corresponding to amino acids 364-449), Er81 (corresponding to amino acids 407-493), and Pea3 (corresponding to amino acids 331416), respectively, by a PCR-based approach, and subcloning into $p C A G G S$ vectors. The FGFR $3 c-\Delta C$ terminus construct and FGFR $3 c-$ $\triangle T M$ construct were prepared by PCR-based cloning of the FGFR $3 c$ coding region (Ornitz and Leder, 1992) containing the extracellular and transmembrane domain (mouse FGFR3c amino acids 1-384) and FGFR3 coding region containing only the extracellular domain (mouse FGFR3c amino acids 1-347), respectively, and subcloning into $p C A G G S$ vectors.

In vivo electroporation. In vivo electroporation was performed as described previously (Saito and Nakatsuji, 2001). Briefly, pregnant mice were deeply anesthetized with sodium pentobarbital (Nembutal, Abbott, North Chicago, IL) at $50 \mathrm{mg} / \mathrm{kg}$ body weight. An incision was made in the abdomen of a pregnant mouse and then the uterus was exposed. The uterine wall was cut, and the embryos covered with extra-embryonic membranes were exposed. DNA solutions ( $5 \mathrm{mg} / \mathrm{ml}$ in PBS with $0.016 \%$ trypan blue to permit visualization of injected solution in the embryos) were injected into the lateral ventricle of E12.5 or E13.5 telencephalon with a glass micropipette under a microscope. Electroporation was performed using tweezer-type electrodes with 5-mm-diameter discs (CUY650P5; Nepa Gene Co., Chiba, Japan). Electronic pulses of $27 \mathrm{~V}$ for E12.5 embryos and $30 \mathrm{~V}$ for E13.5 embryos were charged five times for 50 $\mathrm{msec}$ at $950 \mathrm{msec}$ intervals using a square-pulse electroporator (CUY21EDIT; Nepa Gene Co.). After the procedure, the embryos were placed back into the abdominal cavity of the pregnant mouse. The pregnant mice were maintained for several days, and the embryos at E14.516.5 were subjected to the analyses. The EYFP construct was used to follow the gene-incorporated descendants and adjusted to the same concentration $(1 \mathrm{mg} / \mathrm{ml})$ among the experiments.

To monitor the fluorescence of EYFP, embryos were permitted to develop in vivo for $48-72 \mathrm{hr}$ and fixed by cardiac perfusion with $4 \%$ PFA in $\mathrm{PB}$, and then the whole brains were isolated. The isolated whole brains were postfixed and equilibrated with sucrose as described above. Cryostat coronal sections were made at $20 \mu \mathrm{m}$. Axiovert 200M camera (Carl Zeiss) and Olympus BX61 with a CCD camera DP50 (Olympus, Tokyo, Japan) were used to obtain fluorescent images. Images were processed using Picture Publisher (Micrografx) and Photoshop (Adobe Systems, San Jose, CA) software.

Dual luciferase reporter assay. Dual luciferase reporter assay was performed according to the manufacturer's protocol (Promega, Madison, WI). pGL3-promoter vector (Promega) was used to construct the Pea3Ets transcription factor-responsive reporter, in which five repeats of an optimal Pea3 binding site juxtaposed to SV40 promoter were incorporated as a synthetic promoter, as described previously (Bojovic and Hassell, 2001). pCAGGS vector (Niwa et al., 1991) was used to construct 


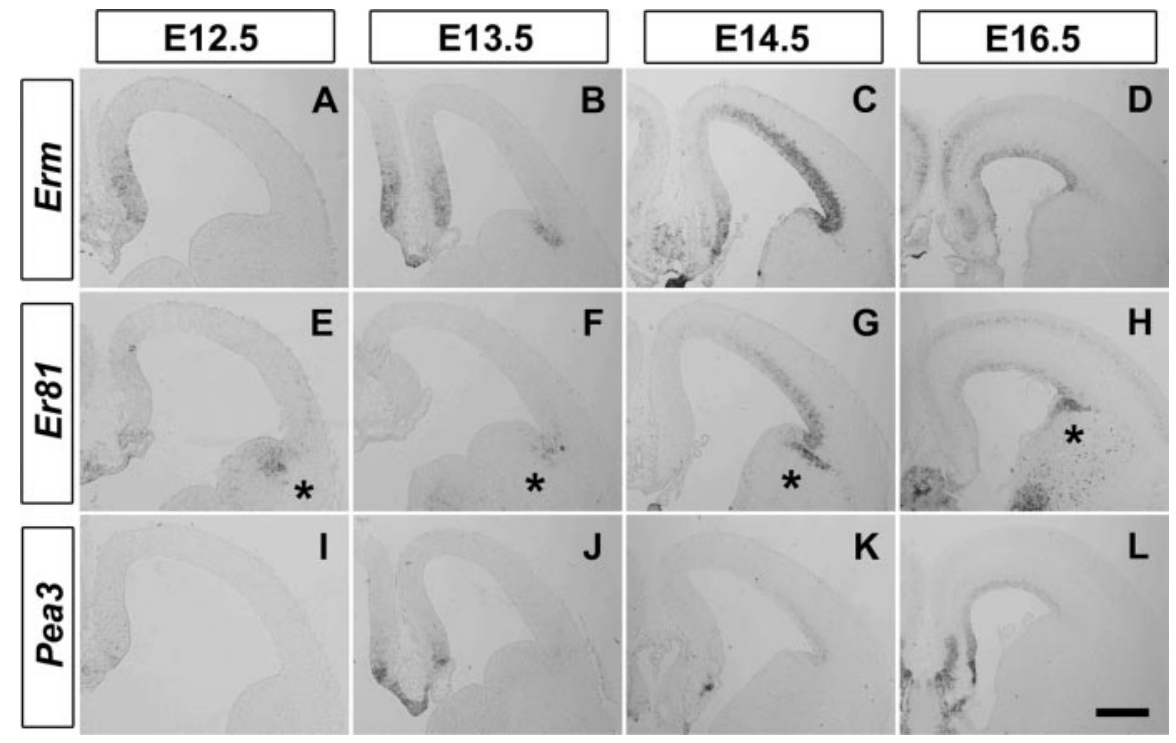

Figure 1. Restricted expression of Pea3-Ets transcription factors in the developing neocortex. Coronal sections of $E 12.5(A, E, I)$, $\mathrm{E} 13.5(B, F, J), \mathrm{E} 14.5(C, G, K)$, and E16.5 $(D, H, L)$ cerebral cortices show patterns of expression of Erm $(A-D)$, Er81 (E-H), and Pea3 $(I-L)$, determined by in situ hybridization. Sections were obtained from an anteroposterior level at which the subcortical ganglionic eminences are located. Asterisks in $E-H$ show Er81 expression in the lateral ganglionic eminence. Erm expression began to be detected in the CP cells located in the deep layers of the medial- dorsal sector of cerebral cortex by E16.5 (D). In addition, Er81 expression began to be detected in a subpopulation of layerV $C P$ neurons by $E 16.5(H)$. Scale bars: $A, E, I, 0.37 \mathrm{~mm} ; B, F, J, 0.41 \mathrm{~mm}$; $C, G, K, 0.48 \mathrm{~mm} ; D, H, L, 0.5 \mathrm{~mm}$

Pea3-Ets transcriptional factor full-length (Ets-FL) and DN forms. After $24 \mathrm{hr}$ of transfection, luciferase activity was measured by extracting COS7 cells transfected with an appropriate amount of each DNA construct. The relative amounts of each DNA construct, Pea3-Ets responsive reporter, Pea3-Ets transcription factor full-length, and $\mathrm{DN}$ form that were transfected together were $45-50,4-5$, and $40-50 \%$, respectively.

\section{Results}

Expression of Pea3-Ets transcription factors in the ventricular zone at the mid to late stages of neocortical development

To begin to address the issue of how Pea3-Ets transcription factors may play roles in cortical development, we first defined the spatial and temporal expression patterns of Erm, Er81, and Pea3 in the mouse cerebral cortex during corticogenesis (Fig. 1A-L).

At E12.5, by which time the generation of deep-layer CP neurons is initiated, the expression of Erm, Er81, and Pea3 was not yet detected at the neocortex (Fig. 1A,E,I). The expression of Erm and Er81, but not Pea3, was first detected at E13.5 in the VZ of the lateral neocortex (Fig. $1 B, F, J$ ). At E14.5, expression of all three transcripts was detected in the neocortical VZ in a lateral-high to dorsal-low gradient (Fig. 1C,G,K). Among the members of the Pea3-Ets transcription factors, Erm exhibited the most prominent expression (Fig. 1C) and Er81 the next (Fig. 1G), whereas Pea3 gave only a faint expression in the neocortical VZ (Fig. $1 \mathrm{~K}$ ). As a continuum of their prominent expressions at the lateral neocortex, the expressions of all the members of the Pea3-Ets transcription factors were detected in the $\mathrm{VZ}$ adjacent to the pallial-subpallial border (Fig. $1 B-D, F-H, K, L$ ). From E16.5 onward, faint expression of all three transcripts persisted uniformly in the neocortical VZ (Fig. 1D, H,L) (data not shown).

These results showed that, in the neocortex, the Pea3-Ets transcription factors Erm, Er81, and Pea3 were expressed by cortical progenitors in the $\mathrm{VZ}$ in an overlapping pattern from E13.5 to 14.5 onward. Given that neocortical neurogenesis initiates at around E10.5-11.5 and declines at around postnatal day $0(\mathrm{P} 0)$ in the mouse neocortex (Polleux et al., 1997; Caviness et al., 2000), these results indicated that expression of Pea3-Ets transcription factors in the neocortical VZ was confined mostly to the mid to late stages of neocortical development when cortical progenitors are dedicated to the generation of intermediate- to upper-layer CP neurons, but not most of the deep-layer CP neurons (Caviness et al., 2000).

\section{Dominant-negative forms of Pea3-Ets transcription factors suppress proper cell body positioning of cortical neurons}

To evaluate functionally the roles played by Pea3-Ets transcription factors expressed by cortical progenitors in the VZ, we prepared DN forms of Pea3-Ets transcription factors in which only the DNAbinding Ets domain was attained. By using an in vitro luciferase reporter assay, we first tested to determine whether each of the DN forms is sufficient to suppress the transcriptional activation mediated by the full-length forms of the Pea3-Ets transcription factors and whether it crossreacts with other Pea3-Ets transcription factors to suppress their transcriptional activities (supplemental Fig. 1, available at www.jneurosci.org as supplemental material). Each DN form was shown to decrease the transcriptional activities achieved by the full-length Pea3-Ets transcription factors to levels achieved by the reporter construct alone. Furthermore, each of the DN forms cross-reacted with all of the other Pea3-Ets transcription factors and suppressed their transcriptional activities to a similar extent, as expected from their sequence similarity among the DNA-binding domains of Pea3-Ets transcription factors (amino acids with $>95 \%$ sequence identity). These results validated the use of these DN forms to suppress transcriptional activities that are mediated by Pea3-Ets transcription factors.

To suppress activities mediated by Pea3-Ets transcription factors in cortical progenitors in the $\mathrm{VZ}$, we used in vivo electroporation-mediated gene transfer to introduce genes into cells located in the VZ. Because cortical neurons located at each layer in the $\mathrm{CP}$ are generated from common progenitors in a temporally precise manner, the in vivo electroporation-mediated gene transfer enabled us to specifically label and follow distinct cortical neurons that are generated from cortical progenitors and located at each layer by controlling the timing of electroporation (H.H. and Y.T., unpublished observations). We electroporated DN forms of Erm (Fig. 2D-F), Er81 (Fig. 2G-I), or Pea3 (Fig. $2 J-L$ ) in conjunction with an EYFP expression construct into VZ cells at the lateral neocortex where the expression of Pea3-Ets transcription factors is detected from E13.5 onward. We permitted the embryos to develop in vivo and followed the geneincorporated descendants at the time when cortical neurons are expected to have left the ventricular zone and have registered tangentially in the CP.

When we electroporated EYFP alone at E13.5 into cells located in the VZ of the lateral neocortex and permitted embryos to develop in vivo until E16.5, most of the EYFP-positive descendants migrated radially from the $\mathrm{VZ}$ of the focal region where the electroporation was conducted (Fig. 2A,B). Most of their cell 
bodies resided in the $\mathrm{CP}(71.4 \pm 5.5 \%)$ (Fig. 2A-C). These EYFP-positive descendants harbored extended neurites, with their cell bodies registered tangentially in the CP (Fig. 2A,B) (data not shown). When we electroporated EYFP together with DN forms of Pea3-Ets transcription factors, most of the descendants that incorporated genes left the VZ (Fig. 2D-L); however, they did not pass beyond the subplate and accumulated underneath it, and accordingly, they localized mainly in the intermediate zone but not in the $\mathrm{CP}$ (percentages of gene-incorporated descendants in the CP were as follows: DNErm: $21.8 \pm 4.9 \%$; DN-Er81:34.7 $\pm 1.9 \%$; DN-Pea3: $33.8 \pm 2.3 \%$ ) at E16.5 (Fig. $2 D$ $L)$. These cells nevertheless expressed neuronal markers, $\beta$-III tubulin, and MAP2 (supplemental Fig. $2 A$, available at www. jneurosci.org as supplemental material) (data not shown), and harbored neurite extensions, suggesting that the generation of cortical neurons was not perturbed even by the expression of DN forms of Pea3-Ets transcription factors. When DN forms of the distantly related members of Ets transcription factors Ets1 (Ets domain amino acids with $64 \%$ sequence similarity with that of Erm) and Elf1 (Ets domain amino acids with $45.3 \%$ sequence similarity with that of Erm), in which only the DNA binding domain was again attained, were electroporated with EYFP, most of the descendants migrated in the $\mathrm{CP}$ to an extent similar to that of the control (DNEts1: $61.1 \pm 1.4 \%$; DN-Elf1: $60.2 \pm 6.5 \%)$ (Fig. 2M-O) (data not shown).

\section{Spatial and temporal correlation} between the expressions of FGFR3 and Pea3-Ets transcription factors in the neocortical VZ

Previous studies have provided evidence that the expression of Pea3-Ets transcription factors in the CNS is controlled by the activities of several classes of cell-extrinsic signaling molecules, including neurotrophin-3, glial cell line-derived neurotrophic factors, Met-activating signals, and FGFs (Raible and Brand, 2001; Roehl and Nusslein-Volhard, 2001; Haase et al., 2002; Helmbacher et al., 2003; Patel et al., 2003). At the caudal levels of the CNS, Pea3-Ets transcription factor expression in the developing motor neurons as well as proprioceptive sensory neurons appears to be induced by cell-extrinsic signals encountered by their axons at the peripheral target area. Pea3-Ets transcription factors appear to be critical for the acquisition of matured phenotypes, including proper cell body positioning and the precise axonal branching at the targets (Lin et al., 1998; Arber et al., 2000; Haase et al., 2002; Livet et al., 2002; Helmbacher et al., 2003; Patel et al., 2003). Our results presented above provided supportive evidence that Pea3-Ets transcription factors were critical for proper cell body positioning of newly generated cortical neurons, which prompted us to examine the identity of plausible environmental signals that might regulate the expression of Pea3-Ets transcription factors and control the proper cell body allocation of cortical neurons.

In a search for candidate receptors of the plausible environmental signals, we determined that FGFR3, which is one of the FGFR subtypes activated dominantly by the FGF8 ligand subfamily (Ford-Perriss et al., 2001; Ornitz and Itoh, 2001), was expressed in a pattern tightly correlated with Pea3-Ets gene expression in the neocortical VZ (Fig. 3). FGFR3 expression was first detected at E12.5, just before the onset of Pea3-Ets transcription factor expression, in the most lateral region of the neocortical VZ adjacent to the pallial-subpallial border where the expression of Pea3-Ets transcription factors was first detected (Figs. $1 A-L, 3 A$ ). At E13.5, the expression of FGFR3 in the VZ extended into a more dorsal region of the neocortex (Fig. $3 B$ ) in a lateral-high to dorsallow gradient. At E14.5, prominent expression of FGFR3c covered nearly the entire region of the neocortical VZ (Fig. 3C). FGFR3 expression was also detected in continuity in the $\mathrm{VZ}$ extending medially into the medial wall of the cerebral hemispheres and laterally into the lateral ganglionic eminence. From E16.5 onward, only faint FGFR3 expression was detected in the neocorti- 


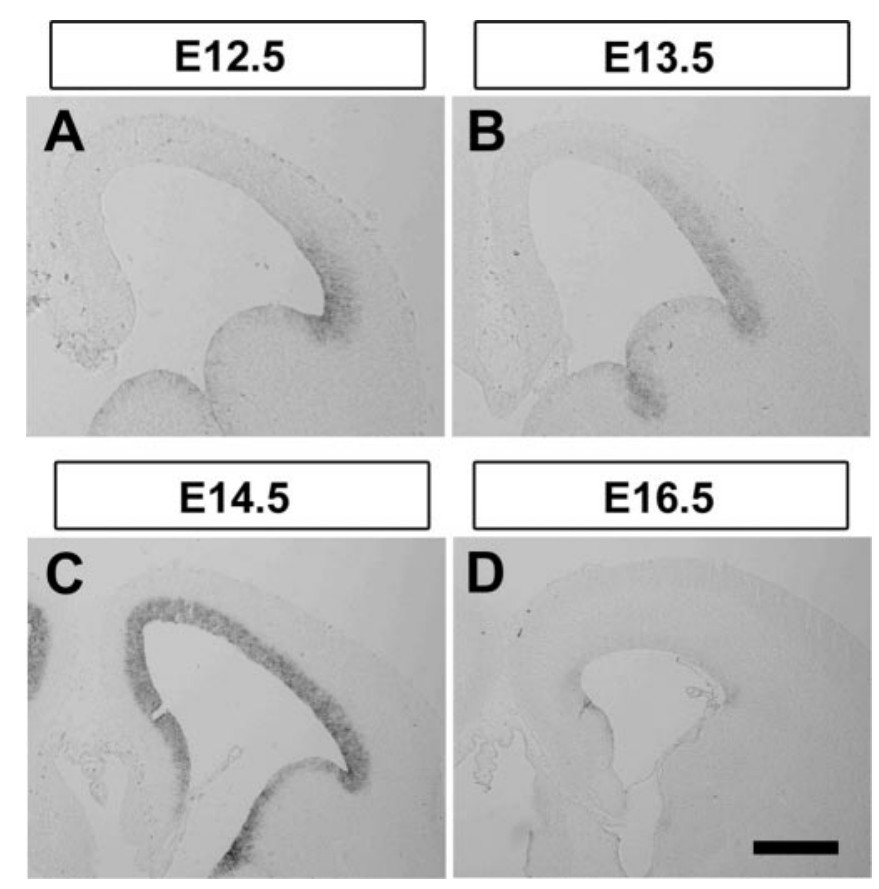

Figure 3. Restricted expression of FGFR3 in cortical progenitors in the ventricular zone of the developing neocortex. Coronal sections of E12.5 (A), E13.5 (B), E14.5 (C), and E16.5 (D) cerebral cortex, showing the expression patterns of FGFR3, determined by in situ hybridization. In situ hybridization analyses revealed the exclusive expression of FGFR3 in the ventricular zone with the spatial and temporal expression patterns that are tightly linked with the expression of Pea3-Ets transcription factors (Fig. 1). Scale bars: $A, 0.37 ; B, 0.41 \mathrm{~mm} ; C, 0.48 \mathrm{~mm} ; D, 0.5 \mathrm{~mm}$.

cal VZ (Fig. 3D) (data not shown). Other FGFRs, FGFR1c and FGFR2c, that preferentially react with FGF8 ligand subfamily members as does FGFR3c also exhibited expressions in the neocortical VZ in a pattern partially overlapping those of Pea3-Ets transcription factors (supplemental Fig. 3, available at www. jneurosci.org as supplemental material). FGFR4 expression was not detected in the neocortical VZ throughout the developmental period (data not shown). Together, these results indicated that there was a tight spatial and temporal correlation between the expression of FGFR3 and that of Pea3-Ets transcription factors in the developing neocortex. The onset of FGFR3 expression appeared to precede those of Pea3-Ets transcription factors in the neocortical VZ, and accordingly, the spatially broader area of FGFR3 expression in the neocortical VZ appeared to contain the areas of Pea3-Ets transcription factor expression. Thus, these observations raised the possibility that FGFR-mediated signals in cortical progenitors might regulate the expression of Pea3-Ets transcription factors and control the proper cell body positioning of their neuronal progeny.

\section{Activation of FGFR is required for proper cell body allocation of cortical neurons and for expression of Pea3-Ets transcription factors}

We first examined whether FGFR-mediated signals in cortical progenitors were critical for newly generated cortical neurons to acquire proper radial migratory behaviors. FGFR dimer formation is known to be a critical step for FGFR activation and signaling, and thus an FGFR form that does not have cytosolic kinase domain inactivates FGFR-mediated signal transduction by forming nonfunctional homodimers as well as heterodimers with other subtypes of FGFRs (Ueno et al., 1992; Plotnikov et al.,
1999). Among the FGFRs that we examined for expressions, FGFR3c gave the most prominent expression at the mid to late phase of corticogenesis (Fig. 3) (supplemental Fig. 3, available at www.jneurosci.org as supplemental material). We thus prepared an FGFR3c- $\Delta$ C terminus form in which the cytosolic domain was deleted. We electroporated $F G F R 3 c-\Delta C$ in lateral neocortical VZ at E12.5 when and where the expression of FGFR3c began to be detected and analyzed the cell body positioning of geneincorporated descendants at E15.5.

When EYFP alone was electroporated at E12.5, most of the EYFP-positive descendants again appeared to migrate properly to cross the subplate and locate their cell bodies in the CP at E15.5 $(67.2 \pm 2.8 \%$ ) (Fig. $4 A-C$ ). In contrast, when FGFR3c- $\Delta C$ was electroporated in conjunction with EYFP, most of the FGFR3c$\Delta C$-incorporated descendants appeared to be located in the intermediate zone and did not pass beyond the subplate $(31.0 \pm$ $3.3 \%$ in the CP) (Fig. $4 D-F$ ), an essentially similar phenotype obtained by electroporating DN forms of Pea3-Ets transcription factors. These results indicate that the proper migration and cell body positioning of newly generated cortical neurons are perturbed by the loss of FGFR-mediated activities in the VZ. FGFR3c- $\Delta$ C-incorporated descendants exhibited several neuronal characters including neurite extensions and the expressions of $\beta$ III-tubulin and MAP2 (supplemental Fig. $2 B$, available at www.jneurosci.org as supplemental material), suggesting that the generation of cortical neurons was not perturbed by the loss of FGFR-mediated signals. Together, these results indicated that activities mediated by FGFRs in cortical progenitors were critical for nascent neuronal progeny to acquire proper migratory behavior and cell body allocation at the mid to late stages of neocortical development.

Next, we attempted to determine whether FGFR-mediated signals might control the expression of Pea3-Ets transcription factors in the neocortical VZ (Fig. 4G-L). To suppress the activities mediated by FGFRs, we prepared an FGFR3c- $\Delta$ TM form, in which only the extracellular domain of FGFR3c was retained (Ornitz and Leder, 1992) to scavenge FGFR-activating environmental signals in the neocortical VZ. Because Pea3 expression was detected only faintly in the neocortical VZ, we analyzed the expressions of Erm and Er81 to evaluate the downregulation of expressions, because these two transcripts can be detected reliably in the neocortical VZ. When we electroporated FGFR3c$\triangle T M$ into the neocortical VZ at E12.5 and analyzed the patterns of Erm and Er81 expressions at E14.5 when the level of their expressions is at its peak during neocortical development, their expressions were downregulated in the neocortical VZ at the region where the electroporation was conducted (Fig. $4 J-L$ ). Prominent expression of Erm and Er81 was detected in the neocortical $\mathrm{VZ}$ on the contralateral side of telencephalic vesicles (Fig. 4G-I).

\section{Spatial and temporal correlation between the expressions of} FGF18 in the CP and Pea3-Ets transcription factors in the VZ The results presented above showed that activation of FGFR in cortical progenitors was critical for conferring proper migratory behavior on nascent cortical progeny. FGFR-mediated signals were also responsible for the expression of Pea3-Ets transcription factors in the neocortical VZ. We next examined whether any endogenous FGF environmental signals might spatially and temporally accompany the expression of Pea3-Ets transcription factors and coordinate activation of FGFRs with expression of Pea3Ets transcription factors at the mid to late phase of neocortical development.

We analyzed the temporal profiles of the expression patterns 
of preferential ligands of FGFR3c, FGF8 ligand subfamily members (Ford-Perriss et al., 2001; Ornitz and Itoh, 2001), FGF8, FGF17, and FGF18, in the developing neocortex (Fig. 5). Among FGF8 ligand subfamily members, prominent expression of FGF18, but not of FGF8 and FGF17, was detected in the CP exclusively in the developing neocortex (Fig. 5A-C) (data not shown). FGF18 was expressed in the CP in a lateral-high to dorsal-low gradient in a pattern similar to $F G F R 3 c$ expression in the VZ at E13.5 (Fig. 5A). At E14.5 when the expression of Pea3-Ets transcription factors is peaked in the VZ, prominent expression of FGF18 was detected exclusively throughout the $\mathrm{CP}$ along nearly the entire dorsolateral axis of the neocortex (Fig. $5 B$ ) in a pattern tightly linked with the expression of Pea3-Ets transcription factors in adjacent VZ. Persistent expression of FGF18 was detected weakly in the entire CP of neocortex from E16.5 onward (Fig. 5C) (data not shown). Together, these results demonstrated that FGF18 was expressed by early-generated cortical neurons after their cell body positioning in the CP, with a pattern that was cohesive with the expression of Pea3-Ets transcription factors by cortical progenitors in the VZ. Thus, these results raised the possibility that FGF18 signals by early-generated cortical neurons located in the CP might temporally coordinate the expression of Pea3-Ets transcription factors in the $\mathrm{VZ}$ at the mid to late phase of neocortical development.

\section{FGF18 temporally coordinates the expressions of Pea3-Ets transcription factors}

To test this possibility, we electroporated FGF18 into the VZ cells at E11.5 and analyzed the expression of Pea3-Ets transcription factors at E13.5 when normally the expression of Pea3-Ets transcription factors is detected only faintly. Misexpression of FGF18 resulted in robust expressions of all Pea3-Ets transcription factors in E13.5 cerebral cortices, suggesting that FGF18 signaling elicits the expression of Pea3-Ets transcription factors and sets up the timing of their expressions (Fig. 5D,E) (data not shown). To further examine whether FGF18 derived from the CP was the environmental signal that contributed to the expression of Pea3Ets transcription factors in the $\mathrm{VZ}$, we analyzed embryonic (E14.5-15.5) FGF18 knock-out mouse cerebral cortices (Ohbayashi et al., 2002). Virtually no expression of all members of the Pea3-Ets transcription factors was detected in the FGF18 (-/-) neocortical VZ, sparing the expressions in other regions of the telencephalon (Fig. 5F-I, insets) (data not shown). These results indicated that FGF18 derived from the early-generated CP neurons acted on the cortical progenitors in a feedback manner and temporally controlled the timing of Pea3-Ets transcription factor expression in the VZ. Together with evidence that activities mediated by FGFRs and Pea3-Ets transcription factors appeared to be critical for conferring proper migratory behavior and cell body allocation on nascent neuronal progeny, these results suggested that a feedback mechanism mediated by FGF18 controls the proper cell body positioning of cortical neurons generated at the mid to late stages of neocortical development (supplemental Fig. 4 , available at www.jneurosci.org as supplemental material).

\section{Discussion}

Our results indicate FGFR-mediated activities in cortical progenitors are critical for nascent neuronal progeny to acquire proper migratory behaviors at the mid to late stages of neocortical development. Our results support the idea that Pea3-Ets transcription factors in cortical progenitors mediate FGFR activities and confer the proper migratory behaviors on neuronal progeny. Moreover, FGF18 expressed by early-generated cortical neurons in the CP controls the expression of Pea3-Ets transcription factors in mid to late phase cortical progenitors at the VZ. Thus, the results of our study lead to the suggestion that a temporally regulated feedback mechanism mediated by FGF signaling underlies the coor- 

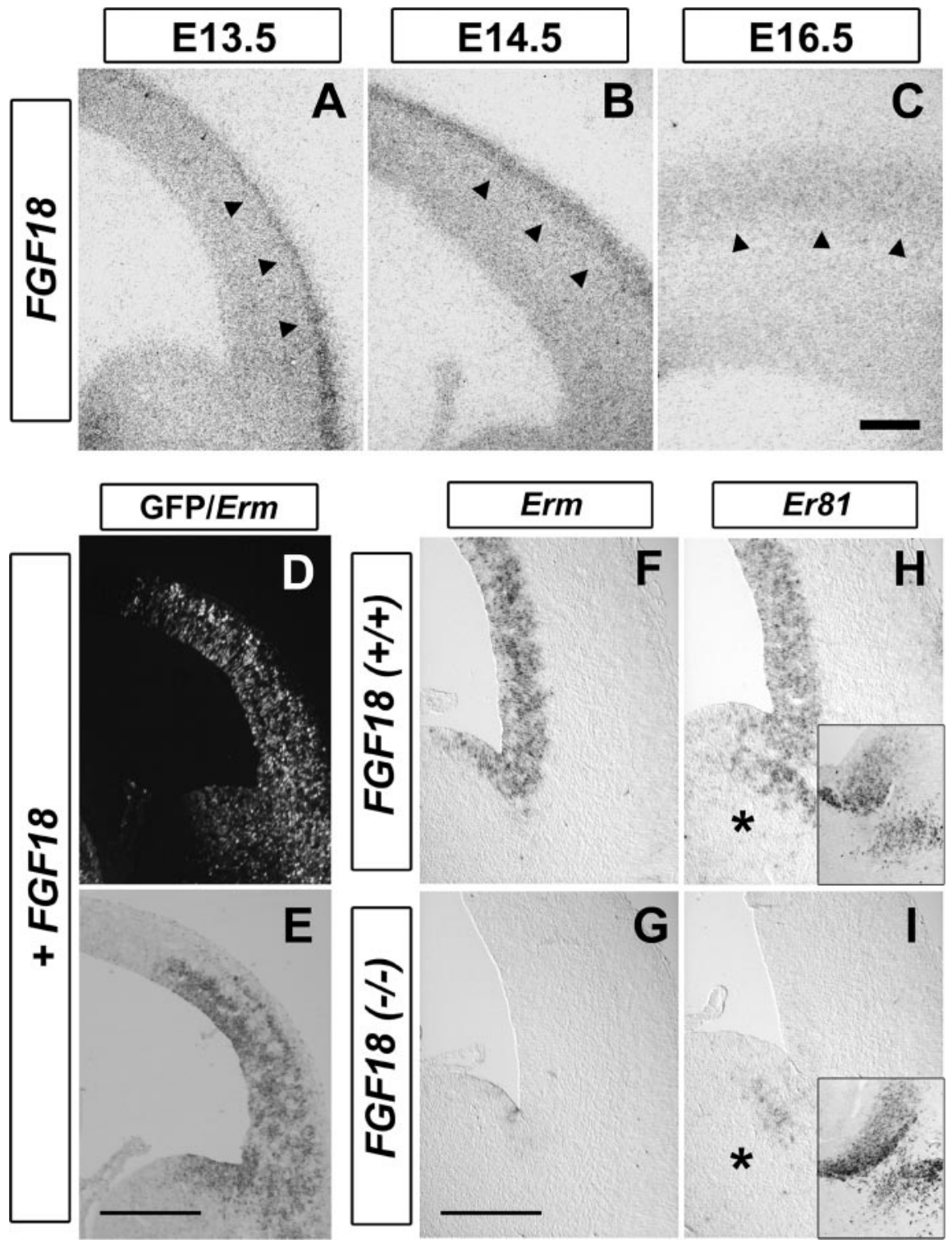

Figure 5. Feedback regulation of Pea3-Ets transcription factor expressions in the VZ by FGF18-mediated signals derived from the CP. A-C, Coronal sections of E13.5, E14.5, and E16.5 cerebral cortices, showing the restricted expression of FGF18 in the CP (arrowheads). Scale bar, $0.3 \mathrm{~mm}$. D, E, Induction of expression of Pea3-Ets transcription factors by FGF18. Misexpression of FGF18, detected by the coexpression of EYFP $(D)$, resulted in an early and ectopic expression of Erm. Images are representatives obtained from more than six electroporated embryos. Scale bar, $0.4 \mathrm{~mm}$. F-I, FGF18 expression in the CP is required for Pea3-Ets transcript expression in the VZ. Coronal sections of E15.5 neocortex derived from FGF18 knock-out mice [FGF18(-/-)] and the wild-type littermates $[F G F 18(+/+)]$ are shown. Asterisks in $\mathrm{H}$ and / show Er81 expression in the lateral ganglionic eminence in both FGF18 knock-out mice and the wild-type littermates. Insets in $\mathrm{H}$ and / show Er81 expression at subcortical ganglionic eminences in both FGF18 knock-out mice and the wild-type littermates. Scale bar, $0.1 \mathrm{~mm}$.

dinated spatial and temporal migratory behaviors of intermediate to upper layer cortical neurons at the mid to late stages of neocortical development and thereby contributes to the laminar patterning of developing neocortex.

\section{FGF signaling as a feedback control of migratory behavior of cortical neurons}

During development of the CNS, temporal patterns of distinct progeny generation from common progenitors are controlled by the intimate interplay between cell-extrinsic and -intrinsic mechanisms (Cepko, 1999; Edlund and Jessell, 1999; Qian et al., 2000). In the developing neocortex, where the major period of cortical neurogenesis precedes the onset of gliogenesis, the temporal con- trol of timing and choice between neuronal and glial developmental pathways in common progenitors appears to be mediated by FGF2-like signals derived from cortical neurons (Qian et al., 1997). These signals are proposed to act on common progenitors in a feedback manner and trigger the switch for the generation of glial cells (Qian et al., 2000). Similar to the developmental programs of neocortical gliogenesis, the acquisition of laminar identities of cortical neurons appears to be controlled by the activities of environmental cues at around the time when progenitor cells leave the final cell cycle to generate cortical neuronal progeny (McConnell and Kaznowski, 1991; Bohner et al., 1997). These studies using heterochronic transplantation of cortical progenitors led to the proposal that a feedback mechanism may operate for the specification of cortical neurons with distinct laminar identities (McConnell, 1995).

Our results suggest that a feedback mechanism mediated by FGF signaling controls the laminar allocation of cortical neurons generated at the mid to late stages of neocortical development. These results imply that early-generated cortical neurons behave as a temporal switch to provide a feedback environmental cue, FGF18. FGF18 acts on progenitors expressing FGFRs and temporally coordinates the expression of Pea3-Ets transcription factors that in turn control the radial migratory behavior and cell body positioning of mid- to late-generated cortical neurons. Thus, FGF signaling operating at the mid to late stages of neocortical development, in addition to its roles in gliogenesis, appears to be involved in the laminar patterning of neocortex. Moreover, our results suggest that, as with the specification of laminar identities of cortical neurons (McConnell and Kaznowski, 1991; McConnell, 1995), the acquisition of distinct migratory behaviors and the laminar allocation of cortical neurons are controlled at least partly in cortical progenitors before the radial migration. Our results do not rule out the possibility that FGF signaling in cortical progenitors is involved in the specification of laminar identities of mid- to late-generated cortical neurons.

In our analyses of the brains of FGF18 knock-out mice, however, we could not detect any migration defects in cortical neurons by the age of $\mathrm{P} 0$, at least, when FGF18 knock-out mice die because of an unknown mechanism (H.H., N.O., S.T., and Y.T., unpublished observations). Nevertheless, our studies of FGFR function in cortical progenitors clearly show an essential role for FGF signaling in the acquisition of proper migratory behaviors and cell body allocation, consistent with a critical role for FGFs and FGFRs in cell migration in many other developmental systems, including invertebrate organisms such as Drosophila and 
Caenorhabditis elegans (Montell, 1999). The precise mechanism of how the lack of FGF18 is compensated remains unknown. Our results show that high levels of FGFR1-3, but not FGFR4, are expressed in the VZ during the period of corticogenesis (Ornitz and Itoh, 2001; Dono, 2003). In the telencephalon, many ligands of FGFRs, including FGF2, FGF7, FGF8, FGF15, and FGF17, are expressed mostly in the VZ, except FGF18, which is expressed in the CP. Thus, in the FGF18 knock-out mice, genetic compensation potentially mediated by these FGFRs and FGFs, together with alternatively spliced isoforms of FGFRs and heparan sulfate proteoglycans, might have resulted in the changes in the requirement of FGFR signal transduction and rescued the radial migration defect. Future studies are needed to determine whether any other FGFs are expressed in the CP and/or VZ, and the potential roles of those FGFs expressed in the developing neocortex in the control of radial migration of cortical neurons generated at midto late-developmental periods. Given the restricted expression of most FGFs in the VZ, however, these studies may reveal rather a cell-autonomous control mechanism mediated by FGF signaling that is operated in the progenitors in the $\mathrm{VZ}$ in an autocrine manner, possibly accompanied by temporal changes in their competence to the FGF signals.

\section{Pea3-Ets transcription factors as mediators of FGF signals in cortical progenitors}

Our approaches using in vivo electroporation-mediated gene transfer to introduce DN forms of Pea3-Ets transcription factors, in which only the DNA-binding Ets domain was retained, appear to suppress the transcriptional activities mediated by Pea3-Ets transcription factors in cortical progenitors, given the suppressive activities of DN forms of Pea3-Ets transcription factors shown in the in vitro studies. Moreover, given that DN forms of distantly related Ets transcription factors do not perturb the proper migratory behaviors of cortical neurons in vivo, our study supports the idea that Pea3-Ets transcription factors, but not other Ets transcription factors, are critical for the acquisition of proper migratory behavior of nascent cortical progeny; however, approaches using these DN forms with $>95 \%$ amino acid sequence similarity did not reveal any potentially distinct activities mediated by each of the members of the Pea3-Ets transcription factors. Moreover, we are left with the question of whether these DN forms of Pea3-Ets transcription factors might have perturbed activities other than those mediated by Pea3-Ets transcription factors.

Nevertheless, our results show that activities mediated by FGFRs are critical for the expression of Pea3-Ets transcription factors in cortical progenitors and for the proper migration of cortical neurons. Thus, these results are consistent with the idea that, in cortical progenitors at the mid to late stages of neocortical development, Pea3-Ets transcription factors act as key mediators that interpret FGF signals to confer proper migratory behavior on nascent neuronal progeny. Furthermore, these results are consistent with the idea that Pea3-Ets transcription factors define a temporal identity of cortical progenitors for the specification of proper migratory behaviors of cortical progeny generated at the mid to late stages of neocortical development. Combinatorial expressions of distinct cell-intrinsic cues, superimposed on the expression of Pea3-Ets transcription factors, in cortical progenitors may further specify their distinct temporal identities for the generation of spatially distinct progeny.

\section{Indirect control of radial migration of cortical neurons by FGF signaling}

Previous studies using molecular genetic approaches in invertebrate organisms such as Drosophila and Caenorhabditis elegans provided insights into the molecular mechanisms of cell migration (Montell, 1999). These studies revealed FGFs and FGFRs as key mediators of cell migration, playing direct roles, for example, in chemoattraction, and/or indirect roles, for example, in the control of cell differentiation. Our results presented above show that FGF signaling is critical for the proper radial migration of cortical neurons. Indeed, the way migratory behaviors of cortical neurons are perturbed by the inhibition of FGF signaling is reminiscent of the phenotypes observed in mouse mutants with cell migration defects (Gupta et al., 2002; Nadarajah and Parnavelas, 2002; Olson and Walsh, 2002), in which cortical neurons cannot pass beyond the subplate and accumulate underneath it. Importantly, however, the expressions of FGFRs and Pea3-Ets transcription factors are confined to cortical progenitors, not detected by radially migrating cortical neurons, suggesting that FGF signaling is involved only indirectly in the control of radial migration through the activities mediated by Pea3-Ets transcription factors that would regulate downstream genes that could play more direct roles in migration.

What are the molecules that are involved more directly in the control of migratory behavior under the control of the FGF signaling pathway? Previous studies in mouse mutants, together with studies in human congenital lissencephaly, have identified several molecules that are critical for the radial migration of cortical neurons (Gleeson and Walsh, 2000; Gupta et al., 2002; Nadarajah and Parnavelas, 2002; Marin and Rubenstein, 2003). At least two distinct modes of radial migration appear to be adopted by cortical neurons, which are controlled by at least two distinct but cooperatively interacting signaling pathways, the reelin- and p35-Cdk5-signaling pathways. Early-generated cortical neurons adopt the nuclear translocation mode, whereas mid- to lategenerated cortical neurons adopt mostly the locomotion mode. Future studies are needed to determine how molecular descendants expressed in migrating neurons under the FGF signaling pathway work together with the reelin- and p35-Cdk5-signaling pathways for the control of microtubule rearrangement and actin cytoskeleton dynamics and eventual laminar formation of neocortex.

\section{References}

Anderson SA, Kaznowski CE, Horn C, Rubenstein JL, McConnell SK (2002) Distinct origins of neocortical projection neurons and interneurons in vivo. Cereb Cortex 12:702-709.

Arber S, Ladle DR, Lin JH, Frank E, Jessell TM (2000) ETS gene Er81 controls the formation of functional connections between group Ia sensory afferents and motor neurons. Cell 101:485-498.

Bohner AP, Akers RM, McConnell SK (1997) Induction of deep layer cortical neurons in vitro. Development 124:915-923.

Bojovic BB, Hassell JA (2001) The PEA3 Ets transcription factor comprises multiple domains that regulate transactivation and DNA binding. J Biol Chem 276:4509-4521.

Brown TA, McKnight SL (1992) Specificities of protein-protein and protein-DNA interaction of GABP alpha and two newly defined Etsrelated proteins. Genes Dev 6:2502-2512.

Caviness Jr VS, Takahashi T, Nowakowski RS (2000) Neuronogenesis and the early events of neocortical histogenesis. In: Mouse brain development (Goffinet AM, Rakic P, eds), pp 107-144. New York: Springer.

Cepko CL (1999) The roles of intrinsic and extrinsic cues and bHLH genes in the determination of retinal cell fates. Curr Opin Neurobiol 9:37-46.

Chotteau-Lelievre A, Desbiens X, Pelczar H, Defossez PA, de Launoit Y (1997) Differential expression patterns of the PEA3 group transcription factors through murine embryonic development. Oncogene 15:937-952. 
Dono R (2003) Fibroblast growth factors as regulators of central nervous system development and function. Am J Physiol Regul Integr Comp Physiol 284:R867-881.

Edlund T, Jessell TM (1999) Progression from extrinsic to intrinsic signaling in cell fate specification: a view from the nervous system. Cell 96:211-224.

Ford-Perriss M, Abud H, Murphy M (2001) Fibroblast growth factors in the developing central nervous system. Clin Exp Pharmacol Physiol 28:493-503.

Gleeson JG, Walsh CA (2000) Neuronal migration disorders: from genetic diseases to developmental mechanisms. Trends Neurosci 23:352-359.

Gupta A, Tsai LH, Wynshaw-Boris A (2002) Life is a journey: a genetic look at neocortical development. Nat Rev Genet 3:342-355.

Haase G, Dessaud E, Garces A, de Bovis B, Birling M, Filippi P, Schmalbruch H, Arber S, deLapeyriere O (2002) GDNF acts through PEA3 to regulate cell body positioning and muscle innervation of specific motor neuron pools. Neuron 35:893-905.

Helmbacher F, Dessaud E, Arber S, de Lapeyriere O, Henderson CE, Klein R, Maina F (2003) Met signaling is required for recruitment of motor neurons to PEA3-positive motor pools. Neuron 39:767-777.

Hu MC, Qiu WR, Wang YP, Hill D, Ring BD, Scully S, Bolon B, DeRose M, Luethy R, Simonet WS, Arakawa T, Danilenko DM (1998) FGF-18, a novel member of the fibroblast growth factor family, stimulates hepatic and intestinal proliferation. Mol Cell Biol 18:6063-6074.

Ishibashi M, Ang SL, Shiota K, Nakanishi S, Kageyama R, Guillemot F (1995) Targeted disruption of mammalian hairy and Enhancer of split homolog-1 (HES-1) leads to up-regulation of neural helix-loop-helix factors, premature neurogenesis, and severe neural tube defects. Genes Dev 9:3136-3148.

Lin JH, Saito T, Anderson DJ, Lance-Jones C, Jessell TM, Arber S (1998) Functionally related motor neuron pool and muscle sensory afferent subtypes defined by coordinate ETS gene expression. Cell 95:393-407.

Livet J, Sigrist M, Stroebel S, De Paola V, Price SR, Henderson CE, Jessell TM, Arber S (2002) ETS gene Pea3 controls the central position and terminal arborization of specific motor neuron pools. Neuron 35:877-892.

Marin O, Rubenstein JL (2003) Cell migration in the forebrain. Annu Rev Neurosci 26:441-483.

McConnell SK (1995) Constructing the cerebral cortex: neurogenesis and fate determination. Neuron 15:761-768.

McConnell SK, Kaznowski CE (1991) Cell cycle dependence of laminar determination in developing neocortex. Science 254:282-285.

Montell DJ (1999) The genetics of cell migration in Drosophila melanogaster and Caenorhabditis elegans development. Development 126:3035-3046.

Nadarajah B, Parnavelas JG (2002) Modes of neuronal migration in the developing cerebral cortex. Nat Rev Neurosci 3:423-432.

Niwa H, Yamamura K, Miyazaki J (1991) Efficient selection for highexpression transfectants with a novel eukaryotic vector. Gene 108:193-200.

Ohbayashi N, Shibayama M, Kurotaki Y, Imanishi M, Fujimori T, Itoh N, Takada S (2002) FGF18 is required for normal cell proliferation and differentiation during osteogenesis and chondrogenesis. Genes Dev 16:870-879.

Olson EC, Walsh CA (2002) Smooth, rough and upside-down neocortical development. Curr Opin Genet Dev 12:320-327.

Ornitz DM, Itoh N (2001) Fibroblast growth factors. Genome Biol 2:reviews3005.1-3005.12.

Ornitz DM, Leder P (1992) Ligand specificity and heparin dependence of fibroblast growth factor receptors 1 and 3. J Biol Chem 267:16305-16311.

Patel TD, Kramer I, Kucera J, Niederkofler V, Jessell TM, Arber S, Snider WD (2003) Peripheral NT3 signaling is required for ETS protein expression and central patterning of proprioceptive sensory afferents. Neuron 38:403-416.

Plotnikov AN, Schlessinger J, Hubbard SR, Mohammadi M (1999) Structural basis for FGF receptor dimerization and activation. Cell 98:641-650.

Polleux F, Dehay C, Kennedy H (1997) The timetable of laminar neurogenesis contributes to the specification of cortical areas in mouse isocortex. J Comp Neurol 385:95-116.

Qian X, Davis AA, Goderie SK, Temple S (1997) FGF2 concentration regulates the generation of neurons and glia from multipotent cortical stem cells. Neuron 18:81-93.

Qian X, Shen Q, Goderie SK, He W, Capela A, Davis AA, Temple S (2000) Timing of CNS cell generation: a programmed sequence of neuron and glial cell production from isolated murine cortical stem cells. Neuron 28:69-80.

Raible F, Brand M (2001) Tight transcriptional control of the ETS domain factors Erm and Pea3 by Fgf signaling during early zebrafish development. Mech Dev 107:105-117.

Roehl H, Nusslein-Volhard C (2001) Zebrafish pea3 and erm are general targets of FGF8 signaling. Curr Biol 11:503-507.

Saito T, Nakatsuji N (2001) Efficient gene transfer into the embryonic mouse brain using in vivo electroporation. Dev Biol 240:237-246.

Takiguchi-Hayashi K, Sekiguchi M, Ashigaki S, Takamatsu M, Hasegawa H, Suzuki-Migishima R, Yokoyama M, Nakanishi S, Tanabe Y (2004) Generation of reelin-positive marginal zone cells from the caudomedial wall of telencephalic vesicles. J Neurosci 24:2286-2295.

Ueno H, Gunn M, Dell K, Tseng Jr A, Williams L (1992) A truncated form of fibroblast growth factor receptor 1 inhibits signal transduction by multiple types of fibroblast growth factor receptor. J Biol Chem 267:1470-1476.

Wasylyk B, Hagman J, Gutierrez-Hartmann A (1998) Ets transcription factors: nuclear effectors of the Ras-MAP-kinase signaling pathway. Trends Biol Sci 23:213-216.

Xin JH, Cowie A, Lachance P, Hassell JA (1992) Molecular cloning and characterization of PEA3, a new member of the Ets oncogene family that is differentially expressed in mouse embryonic cells. Genes Dev 6:481-496.

Xu J, Lawshe A, MacArthur CA, Ornitz DM (1999) Genomic structure, mapping, activity and expression of fibroblast growth factor 17. Mech Dev 83:165-178. 